\title{
ON FINITE GENERATION AND PRESENTABILITY OF SCHÜTZENBERGER PRODUCTS
}

\author{
PETER GALLAGHER and NIK RUŠKUC
}

(Received 25 July 2005; revised 5 May 2006)

Communicated by D. Easdown

\begin{abstract}
The finite generation and presentation of Schützenberger products of semigroups are investigated. A general necessary and sufficient condition is established for finite generation. The Schützenberger product of two groups is finitely presented as an inverse semigroup if and only if the groups are finitely presented, but is not finitely presented as a semigroup unless both groups are finite.
\end{abstract}

2000 Mathematics subject classification: primary 20M05; secondary $20 \mathrm{M} 18$.

\section{Introduction}

This paper concerns Schützenberger products of semigroups and groups, a construction introduced by M.P. Schützenberger [11] in connection with his analysis of monoids without non-trivial subgroups. Subsequently it found other natural applications both in semigroup theory and in language theory; see, for example, $[1,3,5,6]$. In this paper we consider Schützenberger products in the context of finite generation and finite presentability. This strand of research originates in [5], where the authors determine generators and relations that define $S \diamond T$, in the case where $S$ and $T$ are monoids. However, unless $S$ and $T$ are both finite, the set of generators and the set of defining relations for $S \diamond T$ are infinite. There is further work on this topic in [1], in which a more general construction (the Schützenberger product of $n$ groups) is considered and two infinite presentations are exhibited, each of which reflect the structure.

(C) 2007 Australian Mathematical Society 1446-7887/07 \$A2.00+0.00 
Let $S$ and $T$ be semigroups and let $\mathscr{P}_{f}(S \times T)$ denote the set of all finite subsets of the direct product $S \times T$. Define two actions on $\mathscr{P}_{f}(S \times T): S$ acts from the left via $s Q=\{(s p, q):(p, q) \in Q\}$ and $T$ acts from the right via $Q t=\{(p, q t):(p, q) \in Q\}$.

The Schützenberger product $S \diamond T$ is the set $S \times \mathscr{P}_{f}(S \times T) \times T$ with multiplication

$$
\left(s_{1}, Q_{1}, t_{1}\right)\left(s_{2}, Q_{2}, t_{2}\right)=\left(s_{1} s_{2}, s_{1} Q_{2} \cup Q_{1} t_{2}, t_{1} t_{2}\right) .
$$

We immediately observe some basic properties of this construction.

(S1) $S \diamond T$ is a monoid if and only if $S$ and $T$ are monoids, in which case the identity of $S \diamond T$ is $\left(1_{S}, \emptyset, 1_{T}\right)$.

(S2) $S \diamond T$ is finite if and only if $S$ and $T$ are finite.

(S3) $S \diamond T$ is countable if and only if $S$ and $T$ are countable.

(S4) $S \times\{\emptyset\} \times T$ is a subsemigroup of $S \triangleright T$ isomorphic to the direct product $S \times T$. Its complement $(S \diamond T) \backslash(S \times\{\emptyset\} \times T)$ is an ideal of $S \diamond T$.

(S5) $S$ and $T$ are homomorphic images of $S \triangleright T$.

(S6) If $G$ and $H$ are groups then $G \diamond H$ is an inverse semigroup with set of idempotents $\left\{\left(1_{G}, Q, 1_{H}\right): Q \in \mathscr{P}_{f}(G \times H)\right\}$.

The following are our main results.

THEOREM A. Let $S$ and $T$ be semigroups, at least one of which is infinite. The Schützenberger product $S \diamond T$ is finitely generated if and only if the following conditions are satisfied:

(i) $S$ and $T$ are finitely generated;

(ii) $S$ has a unique maximal $\mathscr{R}$-class $R$ and there exists a finite $A \subseteq S$ such that $S=R A$;

(iii) $T$ has a unique maximal $\mathscr{L}$-class $L$ and there exists a finite $B \subseteq T$ such that $T=B L$.

THEOREM B. The Schützenberger product $G \diamond H$ of two groups is finitely presented as an inverse monoid if and only if $G$ and $H$ are finitely presented.

THEOREM C. The Schiitzenberger product $G \triangleright H$ of two groups is finitely presented as a monoid if and only if both $G$ and $H$ are finite.

For standard notions of semigroup theory, and especially for Green's relations $\mathscr{R}$ and $\mathscr{L}$ and natural partial orders defined on them, the reader should consult any standard monograph such as [4]. For the definition and basic properties of inverse semigroups see [7]. The notions relating to presentations will be reviewed in Section 2. The above results will be proved in Sections 3, 5 and 6, respectively, while Section 4 contains some necessary technical material. Where $S$ and $T$ are not groups, the question of finite presentability of $S \diamond T$ remains unsolved. 


\section{Preliminaries}

Denote by $X^{*}$ the free monoid on an alphabet $X$. A pair of words $(u, v) \in X^{*} \times X^{*}$ is called a relation and will be written as $u=v$. For a subset $R \subseteq X^{*} \times X^{*}$ we let $R^{*}$ denote the smallest congruence on $X^{*}$ that contains $R$. We say that $\langle X \mid R\rangle$ presents $S$ as a monoid if $S \cong X^{*} / R^{*}$. We write this as $S=\operatorname{Mon}\langle X \mid R\rangle$. We say that $S$ is finitely presented as a monoid if there are finite $X$ and $R$ such that $S=\operatorname{Mon}\langle X \mid R\rangle$.

For an alphabet $X$ we define a new alphabet $X^{-1}=\left\{x^{-1}: x \in X\right\}$ and denote $X^{ \pm 1}=X \cup X^{-1}$. For a word $w \in\left(X^{ \pm 1}\right)^{*}$, which we write as $w=x_{1}^{\delta_{1}} \ldots x_{n}^{\delta_{n}}$ (with each $\left.x_{i} \in X, \delta_{i} \in\{1,-1\}\right)$, we define $w^{-1}=x_{n}^{-\delta_{n}} \ldots x_{1}^{-\delta_{1}}$. Then the standard inverse monoid relations on $X$ comprise the set

$$
\Re_{X}=\left\{w w^{-1} w=w, w w^{-1} z z^{-1}=z z^{-1} w w^{-1}: w, z \in\left(X^{ \pm 1}\right)^{*}\right\} .
$$

If $S=\operatorname{Mon}\left\langle X^{ \pm 1} \mid R, \Re_{X}\right\rangle$ then $S$ is an inverse monoid, and we use the abbreviation

$$
\operatorname{Inv}\langle X \mid R\rangle=\operatorname{Mon}\left\langle X^{ \pm 1} \mid R, \Re_{X}\right\rangle
$$

and say that $\operatorname{Inv}\langle X \mid R\rangle$ is an inverse monoid presentation of $S$, or that $\operatorname{Inv}\langle X \mid R\rangle$ presents $S$. We say that $S$ is finitely presented as an inverse monoid if there are finite $X$ and $R$ such that $\operatorname{Inv}\langle X \mid R\rangle$ presents $S$.

If an inverse monoid $S$ is finitely presented as a monoid then it is finitely presented as an inverse monoid. Indeed, if $S$ is inverse and $S=\operatorname{Mon}\langle X \mid R\rangle$ then, by adding the standard inverse monoid relations (see (T2) below), $S=\operatorname{Inv}\langle X \mid R\rangle$. The converse does not hold in general: in [10] it is shown that the free inverse monoid is not finitely presented as a monoid (this result also appeared as Theorem IX.4.7 of [7]).

Let us consider a presentation $\operatorname{Mon}\langle X \mid R\rangle$ and two words $w_{1}, w_{2} \in X^{*}$. We write $w_{1} \equiv w_{2}$ if they are identical words. We say that $w_{1}=w_{2}$ holds by one application of a relation in $R$ if we may write $w_{1} \equiv p u_{1} q$ and $w_{2} \equiv p u_{2} q$ where $p, q \in X^{*}$ and $\left(u_{1}=u_{2}\right) \in R$ or $\left(u_{2}=u_{1}\right) \in R$. We say that $w_{1}=w_{2}$ may be deduced from the relations in $R$ if there is a finite sequence of words $v_{1}, \ldots, v_{n} \in X^{*}$ such that $v_{1} \equiv w_{1}$, $v_{n} \equiv w_{2}$ and for each $i=1, \ldots, n-1$ we have that $v_{i}=v_{i+1}$ holds by one application of a relation from $R$. Then elements $s, t \in \operatorname{Mon}\langle X \mid R\rangle$ represented as words over $X$ are equal if and only if this equality can be deduced from the relations $R$.

The following are called Tietze transformations, which may be applied to any monoid presentation $\operatorname{Mon}\langle X \mid R\rangle$.

(T1) Add a symbol $z$ to the list of generators and add $z=w$, for some $w \in X^{*}$, to the list of relations.

(T2) If $w_{1}=w_{2}$, for some $w_{1}, w_{2} \in X^{*}$, may be deduced from the relations $R$, then add this to the list of relations. 
(T3) If there is a symbol $x \in X$ and a relation $x=w$ (for some $\left.w \in(X \backslash\{x\})^{*}\right)$ in the list of relations $R$, then replace $X$ by $X \backslash\{x\}$, remove the relation $x=w$ and replace $x$ by $w$ everywhere else that it occurs in the relations $R$.

(T4) If a relation $w_{1}=w_{2}$, which appears in the list of relations $R$, can be deduced from the other relations, then remove this relation.

Tietze transformations produce equivalent presentations; that is, presentations which define the same semigroup. Moreover, two finite presentations define the same semigroup if and only if there is a finite sequence of Tietze transformations which transforms one into the other.

There are analogous notions of semigroup and inverse semigroup presentations. The basic definitions are given by replacing the free monoid $X^{*}$ by the free semigroup $X^{+}$(which is $X^{*}$ without the empty word) in the above descriptions. Further information on presentations may be found in [9].

\section{Finite generation}

In our examination of the finite generation of the Schützenberger product we will use connections with the direct product (properties (S4) and (S5)). We say that an element $x$ of a semigroup $S$ is indecomposable if there do not exist $y, z \in S$ such that $x=y z$. Then, by [8], the direct product $S \times T$ is finitely generated if and only if $S$ and $T$ are finitely generated and if one is infinite then the other contains no indecomposable elements.

By (S2) above, finite generation of $S \diamond T$ is trivial when $S$ and $T$ are finite. Theorem $A$ deals with the non-trivial situation where at least one of the factors is infinite.

Proof of TheOrem A. $(\Rightarrow)$ Assume that $X$ finitely generates $S \diamond T$. By property (S5) it follows that $S$ and $T$ are finitely generated, so condition (i) holds.

Let $k=1+\max \{|Q|:(x . Q . y) \in X\}$. Then, for arbitrary $s \in S, t \in T$ and $P \in \mathscr{P}_{f}(S \times T)$ with $|P|=k$, there are generators $\left(x_{i}, Q_{i}, y_{i}\right) \in X$ such that

$$
(s, P, t)=\left(x_{1}, Q_{1}, y_{1}\right) \ldots\left(x_{n}, Q_{n}, y_{n}\right) \text {. }
$$

We first note that $n>1$. It is clear that $s=x_{1} \ldots x_{n}$ and $t=y_{1} \ldots y_{n}$, so neither $S$ nor $T$ contain any indecomposable elements.

As $S$ is finitely generated, it has only finitely many maximal $\mathscr{R}$-classes, and every other $\mathscr{R}$-class is below one of these. We let $R_{1}, \ldots R_{l}$ be the maximal $\mathscr{R}$-classes and we select representatives $r_{i} \in R_{i}$ for $i=1, \ldots l$. Now we consider an arbitrary element $s \in S$. There are $u, v \in S$ such that $s=u v$ and there is $i \in\{1, \ldots, l\}$ such that $R_{u} \leq R_{i}$. Hence there is $w \in S^{1}$ such that $u=r_{i} w$, so $w v \in S$ and $s=r_{i} w v$. 
We conclude that for each $s \in S$ there are $\alpha(s) \in\{1, \ldots, l\}$ and $\mu(s) \in S$ such that $s=r_{\alpha(s)} \mu(s)$.

We define the finite set $A=C \cup D$ where

$$
\begin{gathered}
C=\{s \in S:(s, t) \in Q \text { for some }(x, Q, y) \in X \text { and } t \in T\} \\
D=\{\mu(s): s \in C\} .
\end{gathered}
$$

We claim that

$$
S=\left(\bigcup_{i=1}^{l} R_{i}\right) A .
$$

To prove this assertion, we begin by letting $s \in S$ be arbitrary. Further, we pick $t, t_{1} \in T$ and let $P \in \mathscr{P}_{f}(S \times T)$ be a $k$-set containing $(s, t)$. We now consider the triple $\left(r_{1}, P, t_{1}\right)$ and write it as

$$
\left(r_{1}, P, t_{1}\right)=\left(x_{1}, Q_{1}, y_{1}\right) \ldots\left(x_{n}, Q_{n}, y_{n}\right)
$$

a product of generators from $X$. Again we note that $n>1$. Then $r_{1}=x_{1} \ldots x_{n}$, so $x_{1} \ldots x_{j} \in R_{1}$ for every $j=1, \ldots, n$. Equating the middle co-ordinates of (3.2) gives that

$$
P=\bigcup_{j=1}^{n} x_{1} \ldots x_{j-1} Q_{j} y_{j+1} \ldots y_{n} .
$$

Therefore there is $h \in\{1, \ldots, n\}$ and $(a, b) \in Q_{h}$ (so $a \in C$ ) such that

$$
(s, t)=\left(x_{1} \ldots x_{h-1} a, b y_{h+1} \ldots y_{n}\right) .
$$

If $h=1$ then $s=a=r_{\alpha(a)} \mu(a) \in R_{\alpha(s)} A$. If $h>1$ then, clearly, $s \in R_{1} A$. This proves (3.1).

We now see that either $S$ is infinite, in which case, by (3.1), at least one of $R_{1}, \ldots, R_{l}$ is infinite, or else $S$ is finite and $T$ is infinite. In any case, without loss of generality, we may assume that $R_{1} \times T$ is infinite. Let $P=\left\{\left(s_{1}, t_{1}\right), \ldots,\left(s_{k}, t_{k}\right)\right\} \subseteq R_{1} \times T$ be arbitrary of size $k$ and let $t \in T$ be arbitrary. Let us suppose that $l \geq 2$. We consider $\left(r_{2}, P, t\right)$ and write it as another product of generators from $X$ :

$$
\left(r_{2}, P, t\right)=\left(x_{1}, Q_{1}, y_{1}\right) \ldots\left(x_{n}, Q_{n}, y_{n}\right) .
$$

Yet again, note that $n>1$. Further, $Q_{j} \neq \emptyset$ for some $j \geq 2$. For $(a, b) \in Q_{j}$ it follows that $\left(x_{1} \ldots x_{j-1} a, b y_{j+1} \ldots y_{n}\right) \in P$, so there is some $m \in\{1, \ldots, k\}$ with $s_{m}=x_{1} \ldots x_{j-1} a$. Also, $r_{2}=x_{1} \ldots x_{n}$, so $x_{1} \in R_{2}$. It now follows that $R_{1}=R_{s_{m}} \leq R_{x_{1}}=R_{2}$, a contradiction. Hence $l=1$. Therefore $S$ has a unique maximal $\mathscr{R}$-class. Returning now to (3.1), we see that condition (ii) holds. By a symmetric argument, it can be shown that condition (iii) holds as well. 
$(\Leftarrow$ ) Assume that conditions (i), (ii) and (iii) hold. Neither $S$ nor $T$ contain any indecomposable elements (by (ii) and (iii)) and both $S$ and $T$ are finitely generated (by (i)). Hence $S \times T$ is finitely generated and we let $Y$ be a finite generating set for $S \times\{\emptyset\} \times T(\cong S \times T)$. We fix arbitrary $r \in R, l \in L$ and let

$$
X=Y \cup\{(r,\{(a, b)\}, l): a \in A, b \in B\} .
$$

We will complete this part of the proof by showing that $S \diamond T=\langle X\rangle$.

By induction on $|P|$ we will show that $(s, P, t) \in\langle X\rangle$ for all $P \in \mathscr{P}_{f}(S \times T)$ and all $s \in S, t \in T$. The base case $|P|=0$ is obvious as $X$ includes a generating set for $S \times\{\emptyset\} \times T$.

Next we consider the case $|P|=1$, by letting $s, s_{1} \in S$ and $t, t_{1} \in T$ be arbitrary and examining the triple $\left(s,\left\{\left(s_{1}, t_{1}\right)\right\}, t\right)$. As $S=R A$ there are $r_{1} \in R, a \in A$ such that $s_{1}=r_{1} a$. As $R$ is the unique maximal $\mathscr{R}$-class of $S$, there is $u \in S$ such that $s=r_{1} u$, using the fact that $s$ is not indecomposable in the case $s=r_{1}$. Similarly, there is $u_{1} \in S$ such that $u=r u_{1}$. Therefore $s_{1}=r_{1} a$ and $s=r_{1} r u_{1}$. A dual argument shows that there exist $b \in B, l_{1} \in L$ and $v_{1} \in T$ such that $t_{1}=b l_{1}$ and $t=v_{1} l_{1}$. Hence $\left(s,\left\{\left(s_{1}, t_{1}\right)\right\}, t\right)=\left(r_{1}, \emptyset, v_{1}\right)(r,\{(a, b)\}, l)\left(u_{1}, \emptyset, l_{1}\right) \in\langle X\rangle$.

For the inductive step we assume that $\langle X\rangle \supseteq\{(s, P, t):|P| \leq k, s \in S, t \in T\}$ for some positive integer $k$. We let $s, s_{i} \in S . t, t_{i} \in T$ be arbitrary for $i=1, \ldots, k+1$ and consider the triple $\left(s,\left\{\left(s_{1}, t_{1}\right), \ldots\left(s_{k+1}, t_{k+1}\right)\right\}, t\right)$. There exist $r_{1} \in R$ and $x_{1}, x_{2} \in S$ such that $s_{k+1}=r_{1} x_{1}$ and $s=r_{1} x_{2}$. Also, there are $l_{1} \in L$ and $y_{1}, \ldots, y_{k+1} \in T$ such that $t=y_{k+1} l_{1}$ and $t_{i}=y_{i} l_{1}$ for $i=1, \ldots, k$. Then

$$
\begin{aligned}
\left(s,\left\{\left(s_{1}, t_{1}\right), \ldots,\left(s_{k+1}, t_{k+1}\right)\right\}, t\right) & =\left(r_{1} x_{2},\left\{\left(s_{1}, y_{1} l_{1}\right), \ldots\left(s_{k}, y_{k} l_{1}\right),\left(r_{1} x_{1}, t_{k+1}\right)\right\}, y_{k+1} l_{1}\right) \\
& =\left(r_{1},\left\{\left(s_{1}, y_{1}\right), \ldots,\left(s_{k}, y_{k}\right)\right\}, y_{k+1}\right)\left(x_{2},\left\{\left(x_{1}, t_{k+1}\right)\right\}, l_{1}\right)
\end{aligned}
$$

and the claim follows by induction.

From this point we will mainly consider Schützenberger products of groups, so we make the following observation.

COROLlaRY 3.1. Let $G$ and $H$ be groups. The Schützenberger product $G \diamond H$ is finitely generated if and only if $G$ and $H$ are finitely generated. Moreover, $\operatorname{rank}(G \diamond H)=\operatorname{rank}(G \times H)+1$.

PROOF. The first statement of the result follows directly from Theorem A. From the proof of that result, we observe that

$$
G \diamond H=\left\langle G \times\{\emptyset\} \times H,\left(1_{G},\left\{\left(1_{G}, 1_{H}\right)\right\}, 1_{H}\right)\right\},
$$

so $\operatorname{rank}(G \diamond H) \leq \operatorname{rank}(G \times H)+1$.

From the property (S4) it follows that $\operatorname{rank}(G \diamond H)>\operatorname{rank}(G \times H)$, completing the proof. 


\section{Schützenberger products of two groups: an infinite presentation}

In this section we lay foundations for proofs of Theorems $\mathrm{B}$ and $\mathrm{C}$ by obtaining an infinite, but 'nicely behaved', presentation ((4.2) in Theorem 4.2) for the Schützenberger product of two groups. A very similar presentation (which we label here as (4.3)) appears in [1], where it is derived from a presentation for a more general structure.

We show another way to derive it, beginning with the following theorem from [5] and using Tietze transformations.

THEOREM 4.1. If $S=\operatorname{Mon}\left\langle X \mid R_{S}\right\rangle$ and $T=\operatorname{Mon}\left\langle Y \mid R_{T}\right\rangle$ then

$$
\begin{aligned}
S \diamond T= & \operatorname{Mon}\left\langle X, Y, z_{s, t}(s \in S, t \in T)\right| R_{S}, R_{T}, x y=y x, z_{s, t}^{2}=z_{s, t}, z_{s, t} z_{u, v}=z_{u, v} z_{s, t}, \\
& \left.x z_{s, t}=z_{x s, t} x, z_{s, t} y=y z_{s, t y}(x \in X, y \in Y, s, u \in S, t, v \in T)\right\rangle .
\end{aligned}
$$

We now transform presentation (4.1) in the case where the monoids considered are groups $G$ and $H$.

THEOREM 4.2. Let $G$ and $H$ be groups and let $G \times H=\operatorname{Mon}\langle A \mid R\rangle$. Let $z$ be a new letter not in $A$. For each $(g, h) \in G \times H$ we let $w_{g, h} \in A^{*}$ be a fixed word representing $(g, h)$ and let $z_{g, h}=w_{g, h^{-1}} z w_{g^{-1} . h}$. Then

$$
\left\langle A, z \mid R, z^{2}=z, z_{g_{1}, h_{1}} z_{g_{2}, h_{2}}=z_{g_{2}, h_{2}} z_{g_{1}, h_{1}}\left(g_{1}, g_{2} \in G, h_{1}, h_{2} \in H\right)\right\rangle
$$

presents $G \diamond H$ as a monoid.

PROOF. Let $\left\langle X \mid R_{G}\right\rangle$ and $\left\langle Y \mid R_{H}\right\rangle$ present $G$ and $H$, respectively, as monoids. By Theorem 4.1, a presentation for $G \diamond H$ is given by (4.1) with $G$ and $H$ in place of $S$ and $T$ respectively.

For every $g \in G$ we let $w_{g} \in X^{*}$ be a fixed word representing $g$. For every $h \in H$ we let $w_{h} \in Y^{*}$ be a fixed word representing $h$. By repeated application of the relations $x z_{g . h}=z_{x g . h} x$ we can deduce all relations of the form $w_{g_{1}} z_{g . h}=z_{g_{1} g . h} w_{g_{1}}$, so we use the Tietze transformation (T2) to add these to the presentation (4.1). Likewise, from $z_{g . h} y=y z_{g . h y}$ we can deduce all relations of the form $z_{g . h} w_{h_{1}}=w_{h_{1}} z_{g . h h_{1}}$, so we use (T2) to add these to (4.1) as well.

Now, from $w_{g_{1}} z_{g, h}=z_{g_{1}, h} w_{g_{1}}, z_{g, h} w_{h_{1}}=w_{h_{1}} z_{g, h h_{1}}, R_{G}$ and $R_{H}$ we can deduce all relations of the form $w_{g} w_{h^{-1}} z_{1_{G}, 1_{H}} w_{h} w_{g^{-1}}=z_{g, h}$, so we use (T2) to add these to our presentation.

We now use (T3) to remove all $z_{g . h}$, except for $z_{1_{G}, 1_{H}}$, from the generating set, and delete all relations $z_{g, h}=w_{g} w_{h^{-1}} z_{l_{G} .1_{H}} w_{h} w_{g^{-1}}$ from the set of defining relations and 
replace $z_{g, h}$ by the above word in the remaining relations. For the sake of brevity we will rename $z_{\mathfrak{l}_{i .1} I_{H}}$ as $z$. We will also continue to use the symbols $z_{. . h}$ with the understanding that they represent the corresponding words $w_{g} w_{h^{-1}} z w_{h} w_{g^{-1}}$.

From the relations $R_{G}, R_{H}$ and $z^{2}=z$ we can deduce all relations of the form $z_{g, h}^{2}=z_{g . h}$ so we use (T4) to remove these from our presentation, except for $z^{2}=z$.

Similarly, from $R_{G}, R_{H}$ and $x y=y x$ we can deduce all relations of the forms $w_{g,} z_{g . h}=z_{q_{1} . h} w_{g_{1}}$ and $z_{g . h} w_{h_{1}}=w_{h_{1},} z_{g, h h_{1}}$ so we use (T4) to delete these from our presentation. Thus we have transformed the original presentation (4.1) for $G \diamond H$ into

$$
\begin{aligned}
& \langle X, Y, z| R_{G}, R_{H}, x y=y x, z^{2}=z, z_{k_{1}, h_{1}} z_{g_{2}, h_{2}}=z_{g_{2}, h_{2}} z_{k_{1}, h_{1}} \\
& \left.\quad\left(x \in X, y \in Y, g_{1}, g_{2} \in G, h_{1}, h_{2} \in H\right)\right\rangle .
\end{aligned}
$$

Since both $\left\langle X, Y \mid R_{G} . R_{H}, x y=y x(x \in X, y \in Y)\right\rangle$ and $\langle A \mid R\rangle$ define $G \times H$, there is a sequence of Tietze transformations which converts the former into the latter. Applying the same sequence to (4.3) yields the presentation (4.2), as desired.

\section{Finite presentability of $G \diamond H$ as an inverse monoid}

We now use Theorem 4.2 to consider presentations for $G \diamond H$ as an inverse monoid.

THEOREM 5.1. If $G \times H=\operatorname{Mon}\langle A \mid R\rangle$ then $G \diamond H=\operatorname{Inv}\left\langle A, z \mid R \cdot z^{2}=z\right\rangle$.

PROOF. This follows immediately from (4.2) because relations of the form

$$
z_{k_{1}, h_{1}} \bar{z}_{k_{2} . h_{2}}=z_{\xi_{2}, h_{2}} z_{g_{1}, h_{1}}
$$

say that certain idempotents commute, which occurs in any inverse monoid. Thus these relations are consequences of $\Omega_{A \cup: I}$ and may be deleted.

We note, from the above presentation. that $G \diamond H$ is the inverse monoid free product of $G \times H$ and the two-element semilattice. More importantly, Theorem B now readily follows.

PROOF OF THEOREM B. $(\Rightarrow)$ Assume that $G \diamond H$ is finitely presented as an inverse monoid. Again we use property (S4). Corollary 5.4 of [2] states that a subsemigroup with an ideal complement inherits finite presentability (this also appears in [9]), so it follows that $G \times H$ is finitely presented. In turn this implies that $G$ and $H$ are each finitely presented.

$(\Leftarrow)$ If $G$ and $H$ are finitely presented, then $G \times H$ is finitely presented and Theorem 5.1 gives a finite presentation for $G \diamond H$ as an inverse monoid. 


\section{6. (Non)finite presentability of $G \diamond H$ as a monoid}

We return to the question of the finite presentability of $G \diamond H$ as a monoid. Again we use Theorem 4.2.

PROOF OF THEOREM C. Assume that $G$ and $H$ are not both finite but that $G \diamond H$ is finitely presented. Therefore, by the same argument as the proof of Theorem $\mathrm{B}$, but applied to monoid presentations, $G \times H$ is finitely presented as a monoid by $\langle A \mid R\rangle$, say. Remembering the definition of $z_{g . h}$ in Theorem 4.2, we rearrange those relations using $g=g_{1}^{-1} g_{2}$ and $h=h_{1} h_{2}^{-1}$ to see that

$$
\left\langle A, z \mid R, z^{2}=z, w_{g, h} z w_{g^{-1}, h^{-1}} z w_{g, h}=z w_{g, h} z((g, h) \in G \times H)\right\rangle
$$

presents $G \diamond H$ as a monoid. As $G \diamond H$ is finitely presented, the generators of (6.1) and a finite subset of those relations will suffice to define it. Therefore, for some finite $W \subseteq G \times H$, we have

$$
G \diamond H=\operatorname{Mon}\left\langle A, z \mid R, z^{2}=z, w_{g . h} z w_{g^{-1}, h^{-1}} z w_{g . h}=z w_{g . h} z \quad((g, h) \in W)\right\rangle .
$$

We will complete the proof by showing that this is not possible.

Consider a word of the form $z w_{g, h} z$ for some fixed $g \in G, h \in H$ such that $(g, h) \notin W \cup W^{-1} \cup\left\{\left(1_{G}, 1_{H}\right)\right\}$. We claim that any word obtained from $z w_{g, h} z$ by applying the relations from (6.2) is of the form

$$
\alpha_{1} z \alpha_{2} z \ldots \alpha_{r} z \beta z \alpha_{r+1} \ldots z \alpha_{r+1-1} z \alpha_{r+1}
$$

for some $\alpha_{i} \in A^{*}(i=1, \ldots, r+l)$, all of which represent $\left(1_{G}, 1_{H}\right)$, and some $\beta \in A^{*}$ which represents $(g, h)$. Intuitively, a word of the form (6.3) has 'centre' $z \beta z$, which is surrounded by 'redundant' factors which do not change the element of $G \diamond H$ that the word represents. Clearly the word $z w_{g . h} z$ is of this form. We proceed to show that applying any relation from presentation (6.2) to a word of the form (6.3) yields another word of the same form.

A relation from $R$ contains no occurrences of $z$. Hence any application of such a relation is wholly within $\gamma$, where $\gamma=\alpha_{i}$ or $\gamma=\beta$, and it does not change the element of $G \times H$ that $\gamma$ represents.

The result of applying the relation $z^{2}=z$ to a word of the form (6.3) is that, depending on whether the right hand side is substituted for the left or vice versa, the number of occurrences of $z$ is decreased or increased by one and an empty $\alpha_{i}$ is removed or inserted.

We now consider the effect of applying the relation

$$
w_{g_{1}, h_{1}} z w_{g_{1}-1, h_{1}^{-1}} z w_{g_{1}, h_{1}}=z w_{g_{1}, h_{1}} z
$$


for some particular $\left(g_{1}, h_{1}\right) \in W$, to a word of the form (6.3). We first consider the case where this relation is applied by replacing the left hand side by the right. Consider the particular occurrence of $w_{g_{1}, h_{1}} z w_{g_{1}^{-1} \cdot h_{1}^{-1}} z w_{g_{1} \cdot h_{1}}$ as a subword, which is to be replaced by $z w_{g_{1} . h_{1}} z$. If this occurrence is in $\alpha_{i-1} z \alpha_{i} z \alpha_{i+1}$, then $\alpha_{i-1} \equiv c w_{g_{1} . h_{1}}, \alpha_{i} \equiv w_{g_{1}^{-1}, h_{1}^{-1}}$, $\alpha_{i+1} \equiv w_{k_{1}, h_{1}} d$ for some $c, d \in A^{*}$. But $\alpha_{i}$ represents $\left(1_{G}, 1_{H}\right)$, so all of the words

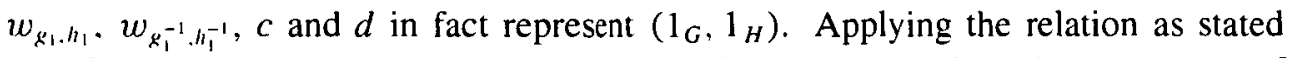
transforms the subword $c w_{g_{1}, h_{1}} z w_{R_{1}^{-1}, h_{1}^{-1}} z w_{g_{1} . h_{1}} d$ into $c z w_{g_{1}, h_{1}} z d$ and leaves the rest of the word unchanged. In particular, the newly obtained word also has the form (6.3). If $w_{g_{1}, h_{1}} z w_{g_{1}^{-1} . h_{1}^{-1}} z w_{g_{1}, h_{1}}$ appears as a subword in $\alpha_{r-1} z \alpha_{r} z \beta$ or $\beta z \alpha_{r+1} z \alpha_{r+2}$, then $w_{\beta_{1} \cdot h_{1}}$ again represents $\left(1_{G}, 1_{H}\right)$ and, by an argument similar to that above, we deduce that applying the relation as stated produces a new word which also has the form (6.3). If $w_{g_{1} . h_{1}} z w_{g_{1}^{-1} \cdot h_{1}^{-1}} z w_{g_{1} \cdot h_{1}}$ is a subword in $\alpha_{r} z \beta z \alpha_{r+1}$, then $\beta \equiv w_{g_{1}^{-1} \cdot h_{1}^{-1}}$. This leads to the contradiction $(g, h)=\left(g_{1}, h_{1}\right)^{-1} \in W^{-1}$, and so this case cannot arise.

We now consider the case where the same relation can be applied by replacing the right hand side by the left hand side. Then $z w_{g_{1} . h_{1}} z$ appears as a subword of (6.3). If this subword is $z \alpha_{m} z$, then $\alpha_{m} \equiv w_{g_{1} . h_{1}}$ and hence $\left(g_{1}, h_{1}\right)=\left(1_{G}, 1_{H}\right)=\left(g_{1}^{-1} \cdot h_{1}^{-1}\right)$. Applying the relation as stated keeps the overall word in the given form. If $z w_{z_{1}, h_{1}} z$ appears in the word as $z \beta z$ then $\beta \equiv w_{k_{1}, h_{1}}$, which leads to the contradiction $(g, h)=\left(g_{1}, h_{1}\right) \in W$. Our claim is shown.

To complete the proof of the theorem we note that since $(g, h) \neq\left(1_{G}, 1_{H}\right)$, the word $w_{g . h} z w_{g^{-1} . h^{-1}} z w_{g . h}$ is not of the form (6.3). Thus the relation

$$
z w_{k . h} z=w_{k . h} z w_{g^{-1} \cdot h^{-1}} z w_{k . h}
$$

holds in $G \diamond H$ but is not a consequence of presentation (6.2), a contradiction.

There is an interesting dichotomy, reminiscent of the free inverse monoid, that the Schützenberger product $G \diamond H$, where $G$ and $H$ are finitely presented infinite groups (or one infinite and one finite), is finitely presented as an inverse monoid but not finitely presented as a monoid.

There remains an obvious unsolved question.

OPEN PROBLEM 6.1. Let $S$ and $T$ be monoids, or even semigroups. Can $S \diamond T$ be finitely presented (without $S$ and $T$ both being finite) and, if so, when?

\section{Acknowledgements}

The authors are grateful to David Easdown and an anonymous referee for their helpful suggestions, which have significantly improved the presentation of the material. 


\section{References}

[1] G. M. S. Gomes. J-E. Pin and H. Sezinando, 'Presentations of the Schützenberger product of $n$ groups', Comm. Algebra to appear.

[2] R. Gray and N. Ruškuc, 'Generators and relations for subsemigroups via boundaries in Cayley graphs', submitted.

[3] J. M. Howie, Automata and languages (Clarendon Press, Oxford, 1991).

[4] - Fundamentals of Semigroup Theory, volume 12 of London Math. Soc. Monogr. Ser. (Clarendon Press. New York, 1995).

[5] J. M. Howie and N. Ruškuc. 'Constructions and presentations for monoids', Comm. Algebra 22 (1994). 6209-6224.

[6] S. W. Margolis and J-E. Pin. 'Expansions, free inverse semigroups and Schützenberger product'. J. Algebra 110 (1987), 298-305.

[7] M. Petrich, Inverse semigroups (John Wiley and Sons Publication, 1984).

[8] E. F. Robertson. N. Ruškuc and J. Wiegold, 'Generators and relations of direct products of semigroups'. Trans. Amer. Math. Soc. 350 (1998). 2665-2685.

[9] N. Ruškuc, Semigroup presentations (Ph.D. Thesis, University of St Andrews, 1995).

[10] B. M. Schein. 'Free inverse semigroups are not finitely presented', Acta Math. Acad. Scient. Hung. 26 (1975), 41-52.

[11] M. P. Schützenberger. 'On finite monoids having only trivial subgroups', Information and Control 8 (1965), 190-194.

School of Mathematics and Statistics

University of St Andrews

St Andrews

Scotland, UK

e-mail: Peterg@mcs.st-and.ac.uk

e-mail: nik@mcs.st-and.ac.uk 
